\title{
Technology and application of operation control of DC distribution network
}

\author{
Kuo Tan ${ }^{1,2,3}$, Hai $\mathrm{Wu}^{1,2,3}$, Guo Hu${ }^{1,2,3}$, Qi Huang ${ }^{1,2,3}$ \\ ${ }^{1}$ NARI Technology Co., Ltd., Nanjing 211000 \\ ${ }^{2}$ NARI Group Corporation (State Grid Electric Power Research Institute), Nanjing 211000 \\ ${ }^{3}$ State Key Laboratory of Smart Grid Protection and Control, Nanjing 211000)
}

\begin{abstract}
DC distribution network consists of distributed generation, energy storage, load and AC distribution network, With the rapid development of DC distribution network and the increasing proportion of DC load, the operation mode of DC distribution network is more flexible. Different from the traditional AC distribution network, the traditional AC distribution network has a single operation mode, while the DC distribution network can operate in both parallel mode and island mode. Therefore, a control method suitable for different operation modes of DC distribution network is urgently needed. In this paper, a control method about loop closing and loop opening of DC distribution network is proposed. When receiving the command of loop closing or loop opening from the master station or local monitoring system, the loop closing control is judged by the switch state, fault state and operation state of converter. It solves the problem that the traditional AC distribution network control strategy is not applicable in DC distribution network, it realizes the smooth switching of DC distribution network closed loop operation and opened loop operation. This method has been used in the DC coordinated control device and successfully applied in the national key research and development plan of Suzhou active distribution network.
\end{abstract}

\section{Introduction}

With the change of power generation, transmission, distribution and utilization, many inherent defects of the traditional AC distribution system are constantly exposed. On the one hand, the radial AC partition power supply can not achieve closed loop operation, it can not meet the demand of high-density load area for power supply capacity, and the traditional AC system can improve the power supply capacity and cause shortcircuit current exceeding the standard. On the other hand, with the rapid development of distributed generation, the increase of the proportion of DC load, the increase of the use of energy storage devices and the improvement of power supply quality requirements of sensitive loads, the $\mathrm{AC}$ form no longer has absolute advantages in terms of efficiency, reliability or power quality ${ }^{[1-2]}$.

DC distribution network consists of distributed generation, energy storage, load and AC distribution network. With the continuous increase of DC load, the research of DC distribution network has been developed rapidly. Compared with traditional AC distribution network, DC distribution network has many advantages, such as less conversion times, high efficiency, low cost, simple control structure and so on ${ }^{[3-4]}$. Compared with AC distribution network, the operation mode of DC distribution network is more flexible. DC distribution network can operate in grid connected mode and island mode, and converter also has a variety of operation states.
Different from the traditional AC distribution network, the traditional AC distribution network has a single operation mode, while the DC distribution network can operate in both parallel mode and island mode. Therefore, a control method suitable for different operation modes of DC distribution network is urgently needed ${ }^{[5-6]}$. In this paper, a control method about loop closing and loop opening suitable for DC distribution network is proposed, which overcomes the shortcomings of the existing technology, it solves the problem that the traditional AC distribution network loop closing control strategy is not applicable in DC distribution network, and it realizes the smooth switching of DC distribution network loop closing and loop opening process.

\section{Control method}

\subsection{System structure and operation characteristics of DC distribution network}

As shown in Figure 1, a typical medium voltage DC distribution network is generally composed of DC converter, DC switch and a series of DC loads (such as DC charging pile, industrial DC motor, server load, DC office load, DC home appliances, etc.). The network adopts the "ring type at both ends" structure, and the two buses connected by a contact switch, each bus connected

\footnotetext{
* Corresponding author: 78939647@qq.com
} 
the $\mathrm{AC} / \mathrm{DC}$ converter station. Each load or distributed converter are connected to the nearby DC bus.

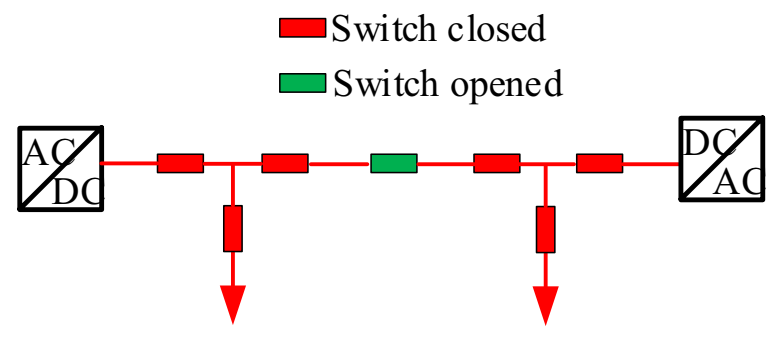

Figure 1 typical DC distribution network structure

Under the normal operation mode, the contact switch in is in the open state, and each converter with part of the DC load works independently. In order to meet the requirements of stable operation, reducing consumption, maintenance and restoring the original operation mode after fault treatment, the DC distribution network should also consider the closed-loop operation, load transfer and other conditions ${ }^{[7-8]}$.

\subsection{Control method of closed loop}

The control device can collect the switch state, fault status and operation status of the connection converter of the closed loop point switch in real time, When it receives the closed loop control command from the master station or local monitoring system, the control device begins to judge the state of contact switch, fault status and operation status of DC distribution network, it can judge whether the closed loop operation can be carried out through the following logic. The operation process is shown in Figure 2, and the operation steps are as follows:

- The control device receives the closing control command from the master station or local monitoring system, and start the closing control process.

- The control device judges whether the state of the contact switch is closed. If it is closed already, the closing control fails.

- The control device judges whether the contact switch is in the fault state. If it is in the fault state already, the closing control fails.

- If one side of the contact switch is connected with a converter and the converter is operated as a voltage source, the closing command will be executed directly.

- If both sides of the contact switch are connected with converters and all converters are operated as a voltage source,

- The control device will send the command of switching from voltage source to power source.

- If the standby converter is changed from voltage source operation to power source operation.

- The synchronous voltage meets the closing requirement, the closing command will be executed directly and control success, Otherwise, if the voltages is not synchronous.

- The control device waits until voltages is synchronous, if it is time out, the closing control fails.

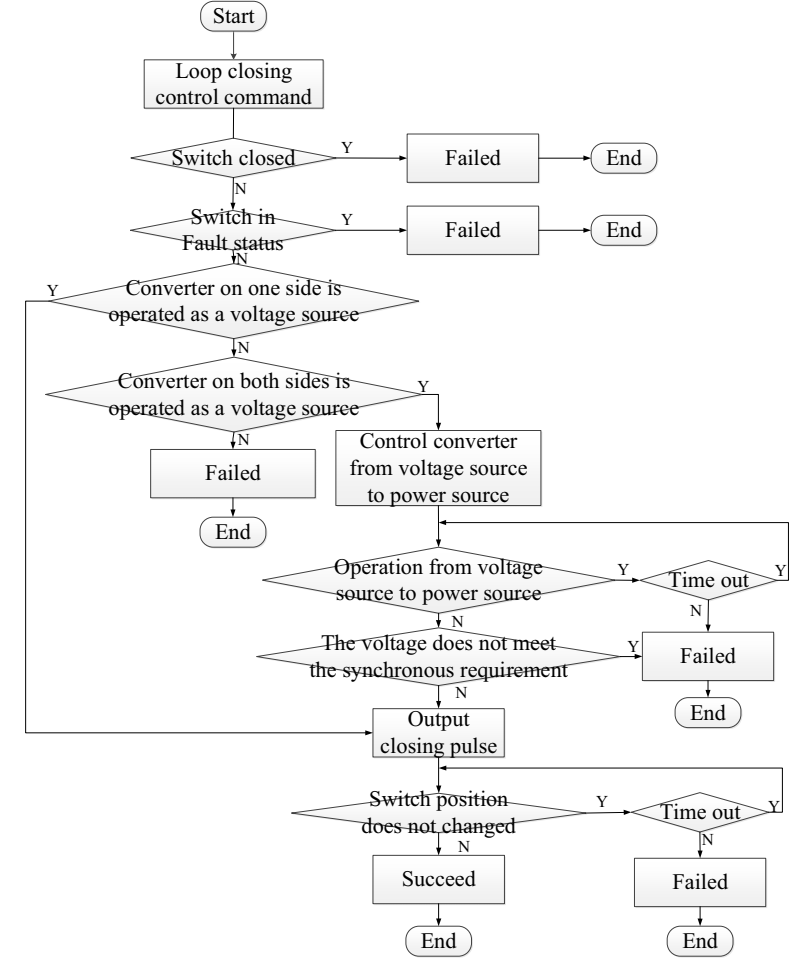

Figure 2 loop closing control logic diagram

\subsection{Control method of opened loop}

Similarly, the control device can collect the switch state, fault status and operation status of the connection converter of the closed loop point switch in real time. when the control device receives the opened control command from the master station or local monitoring system. the control device begins to judge the state of contact switch, fault status and operation status of DC distribution network. It can judge whether the opened operation can be carried out through the following logic. The operation process is shown in Figure 3, The operation steps are as follows:

- The control device receives the opening control command from the master station or local monitoring system, and start the closing control process.

- The control device judges whether the state of the contact switch is opened. If it is opened already, the opening control fails.

- The control device judges whether the contact switch is in the fault state. If it is in the fault state already, the opening control fails.

- If two converters are connected on one side of the contact switch, and one converter is operated as a voltage source, the other one is operated as a power source. , the opening command will be executed directly.

- After the switch is successfully opened, the control device will send the command of switching from power source to voltage source.

- If operation mode is changed from power source to voltage source of the standby converter, it meas control success. Otherwise, the opening control fails. 


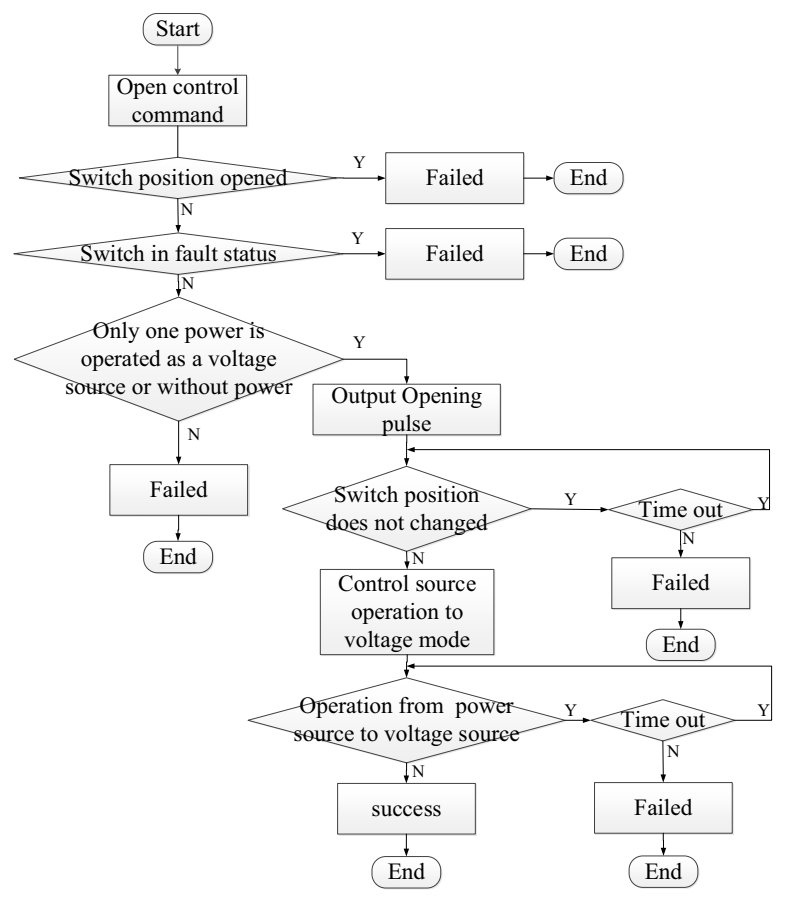

Fig. 3 loop control logic diagram

\section{Achievement application and technical advantages}

\subsection{Project introduction}

"Key technology and application project of DC distribution network" as a national key research and development project, there are three voltage levels in this DC distribution network, it includes DC $10 \mathrm{kV}, \mathrm{DC} 750 \mathrm{~V}$ and DC375V. It is the first DC distribution network project with multiple voltage levels in China, which can meet the needs of high reliable power supply, green energy consumption and DC loads. The project consists of three parts: main grid side, distribution side and user side. The main grid side includes two DC central stations (Pangdong central station and Jiuli central station) in Wujiang Economic and Technological Development Zone, and the distribution side includes seven distribution rooms and three photovoltaic booster stations. The system architecture is shown in Figure 4:

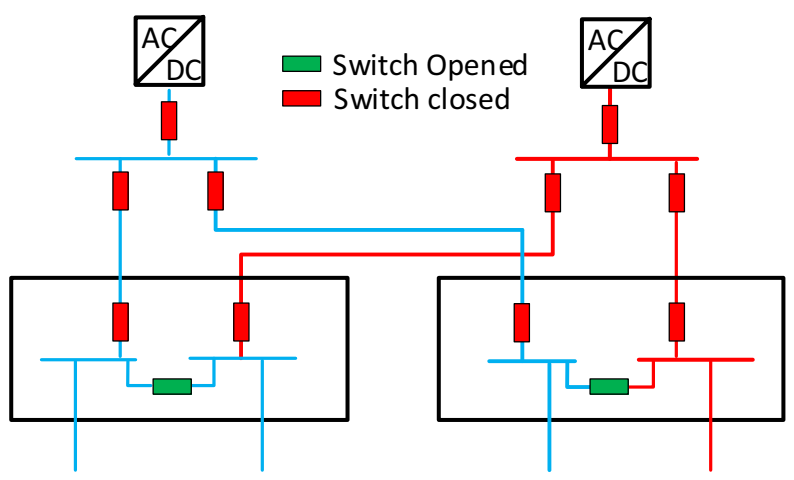

Figure 4 Schematic diagram of system architecture
DC distribution network is an important supporting link of the future energy internet. The multi voltage level DC distribution network can meet the needs of different customers. It realizes the friendly interconnection of various DC power sources, loads and energy storage. The DC distribution network can make Suzhou power grid more "intelligent", it realizes the efficient access of DC power sources and loads such as photovoltaic and electric vehicles. It can also reduce the volume of photovoltaic grid connected devices effectively, and improve the energy efficiency of household appliances.

\subsection{System scheme}

The control of medium voltage side DC distribution network is divided into regional operation control and coordinated control. According to the system operation mode and functional requirements, through the strategy configuration of regional operation control and coordinated control. Through The process monitoring and system reconfiguration of tie line power control, ring closing and ring breaking operation, it realizes operation mode switching smoothly of DC distribution network.

The communication of medium voltage DC distribution network is based on the GOOSE mechanism of IEC61850. Through the communication networking of $\mathrm{AC} / \mathrm{DC}$ converter, DC / DC converter, DC protection and process layer coordination control equipment, it realizes the operation mode switching friendly of DC distribution network with accurate timing control.

From opening loop to closing loop: when two AC / DC converters in DC distribution network operate separately in voltage source mode. when the control device receives the ring opening command from the master station, The control device sends the ring closing command after adjustment to accomplish operation mode switching smoothly.

From closing loop to opening loop: when two AC / DC converters in DC distribution network operate in parallel in master-slave mode. when the control device receives the ring opening command from the master station, The control device sends the ring opening command after adjustment to accomplish operation mode switching smoothly.

\subsection{Achievement and advantages}

The control device of DC distribution network based on this control method has been successfully applied to Suzhou DC distribution network, and achieved effect. It solves the problem that the traditional closed loop control strategy of AC distribution network is not applicable to DC distribution network. It avoids the problem that multiple voltage sources operating caused by directly sending command at the same time. It realizes smooth switch of closing and opening of DC distribution network.

There are many kinds of operation modes in DC distribution network. The traditional control strategy of AC distribution network is not applicable in DC distribution network. The control device can collect the 
status of operation in real time. After sending the pulse command of closing and opening pulse, the control device controls converter to switch the operation mode with real time control strategy, which solves the problem that all converters which operated at voltage mode sources mode at the same time caused sending the pulse command of closing and opening directly.

Compared with the existing technologies, the advantages of the loop closing and loop opening control methods proposed in this paper are shown in Table 1:

Table 1. Comparison table of loop closing control methods and loop openning control methods

\begin{tabular}{|l|c|c|}
\hline & $\begin{array}{l}\text { The control method } \\
\text { of closing loop and } \\
\text { opening loop for DC } \\
\text { distribution network } \\
\text { in this paper }\end{array}$ & $\begin{array}{l}\text { The control method } \\
\text { of loop closing and } \\
\text { loop opening in } \\
\text { traditional AC } \\
\text { distribution network }\end{array}$ \\
\hline $\begin{array}{l}\text { Multi } \\
\text { power } \\
\text { access }\end{array}$ & Supported & Unsupported \\
\hline $\begin{array}{l}\text { Switching } \\
\text { of power } \\
\text { operation } \\
\text { mode }\end{array}$ & Supported & Unsupported \\
\hline $\begin{array}{l}\text { switching } \\
\text { time of } \\
\text { Loop closed }\end{array}$ & Millisecond & Minute \\
\hline $\begin{array}{l}\text { Switching } \\
\text { time of loop } \\
\text { opened }\end{array}$ & Millisecond & Minute \\
\hline
\end{tabular}

\section{Conclusion}

This paper provides a method for loop closing and loop opening control in DC distribution network. This method based on the goose communication protocol of IEC61850, the control device collects operation status of DC distribution network in real time. The operation status includes converter operation mode, switch state and fault state. Through the closed loop and loop time sequence control strategy, It avoids the problem that all converters which operated at voltage mode sources mode at the same time caused sending the pulse command of closing and opening directly. It realizes the smooth switching of closing loop and opening loop in DC distribution network.

\section{References}

1. DUAN Jiandong, LI Zainana, WEI Zhaoyang, et al. A line accelerated protection scheme of flexible MVDC distribution system based on transient current derivative $[\mathrm{J}]$. Electrical Power System Research, 2020, 183: 106269

2. DAI Zhihui, Ge Hongbo, CHEN Bingyan, et al. Line protection schemes for flexible medium voltage DC distribution networks [J] . Automation of Electric Power Systems, 2017, 41 (17) : 7886
3. DAI Zhihui, Ge Hongbo, CHEN Bingyan, et al. Line protection schemes for flexible medium voltage DC distribution networks $[\mathrm{J}]$. Automation of Electric Power Systems, 2017, 41 (17) : 7886

4. WU W, CHEN Y, LUO A, et al. A virtual inertia control strategy for DC microgrids analogized with virtual synchronous machines [ J ] . IEEE Transactions on Industrial Electronics, 2017, 64 (7) : 6005-6016.

5. ZHENG Huan, JIANG Daozhuo, DU Yi. Economic comparison of AC and DC distribution system [J] . Power System Technology, 2013, 37 (12) : 3368-3374.

6. ZHU Ruike, WANG Yuhong, LI Xingyuan, etal . An adaptive DC voltage droop control strategy for the VSCG MTDC system[J] . Automation of Electric Power Systems, 2105, 39(4): 63-68. DOI: 10.7500 / AEPS20131010007.

7. CHEN Hairong, XU Zheng. A novel DC voltage control strategy for VSC based multi terminal HVDC system [J] . Automation of Electric Power Systems,2006,30(19):28-33.

8. WU W, CHEN Y, LUO A, et al. A virtual inertia control strategy for DC microgrids analogized with virtual synchronous machines $[\mathrm{J}]$. IEEE Transactions on Industrial Electronics, 2017, 64 (7) : 6005-6016.

9. GUAN M, XU Z, CHEN H . Control and modulation strategies for modular multilevel converter based HVDC system[C]//IECON2011G37 the Annual Conference on IEEE Industrial Electronics Society, November 7-10,2011, Mel bourne, VIC, Australia:849-854. 